\title{
Withdrawn: 'Rubenstein-Taybi Syndrome with bilateral absence of patella: a case report' [Braz. J. Biol. Sci. (2016), vol. 3, no. 5, p. 227-230]
}

\section{Sedigheh Ganji-Harsini', Sepide Seydi ${ }^{1}$, Ali Ghanbari ${ }^{1 *}$, Mostafa Sedighi $^{3}$, Mazyar Khazali ${ }^{3}$ and Mahsa Falahi ${ }^{2, * *}$}

${ }^{1}$ Fertility Infertility Research Center, Kermanshah University of medical sciences, Kermanshah, Iran.*Email: aghanbari@kums.ac.ir.

2Student Research Committee, Kermanshah University of medical sciences, Kermanshah, Iran.**Email: falahi.mahsa@gmail.com.

${ }^{3}$ Department of pediatrics, Kermanshah University of medical sciences, Kermanshah, Iran.

This article has been withdrawn at the request of the authors. The Editor-in-Chief and Publisher apologizes for any inconvenience this may cause.

This is a notice of withdraw of the article: "Rubenstein-Taybi Syndrome with bilateral absence of patella: a case report" by Sedigheh Ganji-Harsini, Sepide Seydi, Ali Ghanbari, Mostafa Sedighi, Mazyar Khazali and Mahsa Falahi published in the Braz. J. Biol. Sci. (2016), vol. 3, no. 5, p. 227-230, at the request of the correspondent author.

Due to accidentally submission and the author's later request (see above) the Editor-in-Chief of the Brazilian Journal of Biological Sciences decide to retract the paper.

The publisher apologizes for any inconvenience this may cause.

"Dear editor

Unfortunately, we regret continuing publication process of the paper because of our internal rules that allow us only to present our works in ISI listed journals. Seeing IF $=2.5$, this mistake was occurred by me and this condition is not related to nice Brazilian journal of biological sciences. Thus we could not fill the permission form. Please forgive us and withdraw the paper from further publication process.

Best wishes

Dr. Ali Ghanbari

Correspond author"
Received July 19, 2016

Accepted

July 25, 2016

Released

December 31, 2016

Open Acess Full Text Article

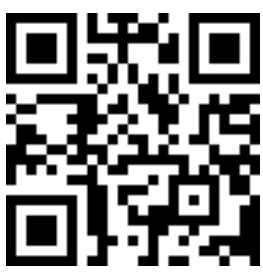

